\title{
Multifocal primary melanoma of the cervical spinal cord successfully treated by tumorectomy: a case report
}

\author{
Takahito Iga ${ }^{1}$ Akio Iwanami ${ }^{2} \cdot$ Takeru Funakoshi $^{3} \cdot$ Shuji Mikami $^{4} \cdot$ Osahiko Tsuji $^{1}$. Narihito Nagoshi ${ }^{1}$. \\ Eijiro Okada ${ }^{1} \cdot$ Nobuyuki Fujita $^{1} \cdot$ Mitsuru Yagi $^{1} \cdot$ Kota Watanabe $^{1} \cdot$ Masaya Nakamura $^{1} \cdot$ Morio Matsumoto $^{1}$
}

Received: 8 October 2017 / Revised: 15 February 2018 / Accepted: 22 February 2018

(c) International Spinal Cord Society 2018

\begin{abstract}
Introduction Multifocal primary melanoma of the spine is extremely rare. Here, we report a case of multifocal primary melanoma of the cervical spinal cord.

Case presentation A 39-year-old man presented with a 1-year history of numbness of the upper extremities and back. Magnetic resonance imaging of the cervical spine suggested multifocal intradural extramedullary tumors at levels $\mathrm{C} 2$ and C4-C5. A diagnostic biopsy revealed a pathological diagnosis of malignant melanoma. Tumorectomy was then performed. The numbness in the patient's upper extremities improved, and he was discharged. As of 2 years after surgery, no signs of local or systemic recurrence have been noted.

Discussion Primary melanoma of the spinal cord usually carries a poor prognosis. However, in this case, the outcome following tumorectomy has been highly favorable. We speculate that this is because the MIB-1 labeling index of the tumors was less than $1 \%$.
\end{abstract}

\section{Introduction}

Primary melanoma of the spinal cord is a rare tumor, and multiple localized melanoma is even less common. Since the first report by Hirschberg in 1906, 60 cases of primary spinal melanoma have been reported in the English literature [1]. To date, there have been only four previous reports of multifocal primary melanocytic tumors [2].

Here, we describe the clinical features and treatment of a patient with multifocal primary melanoma of the cervical spinal cord.

Akio Iwanami

akioiwanami@gmail.com

1 Department of Orthopaedic Surgery, Keio University School of Medicine, Tokyo, Japan

2 Department of Orthopaedic Surgery, Spine Center, Koga Hospital, Ibaraki, Japan

3 Department of Dermatology, Keio University School of Medicine, Tokyo, Japan

4 Department of Pathology, Keio University School of Medicine, Tokyo, Japan

\section{Case presentation}

A healthy 39-year-old man noticed numbness in his upper extremities and back. He visited his primary care physician and was treated conservatively over a period of 1 year. Because he did not experience any improvement, he underwent magnetic resonance imaging (MRI) of the cervical spine. The MRI findings suggested multifocal intradural extramedullary tumors at C1-2, C3-4, and C4-5, and he was referred to our hospital.

The patient had no relevant medical or family history and no history of skin excision surgery. Neurological examination on admission revealed numbness and sensory dysfunction at the C5-Th8 dermatome and motor weakness of the left upper extremities. He was classified as American Spinal Injury Association Impairment Scale (AIS) D at the C5 level. There was no bladder dysfunction or gait disorder. Deep tendon reflexes were normal. MRI revealed several dura-based extramedullary solid tumors ventral to the C1-2 spinal cord and dorsal to the C3-4 and C4-C5 spinal cord, causing compression of the cord without cord signal changes. The tumors appeared hyperintense on T1-weighted images and iso- and hypointense on T2-weighted images, and were strongly enhanced by gadolinium on T1-weighted images, suggesting malignant melanoma (Fig. 1a-c). No 

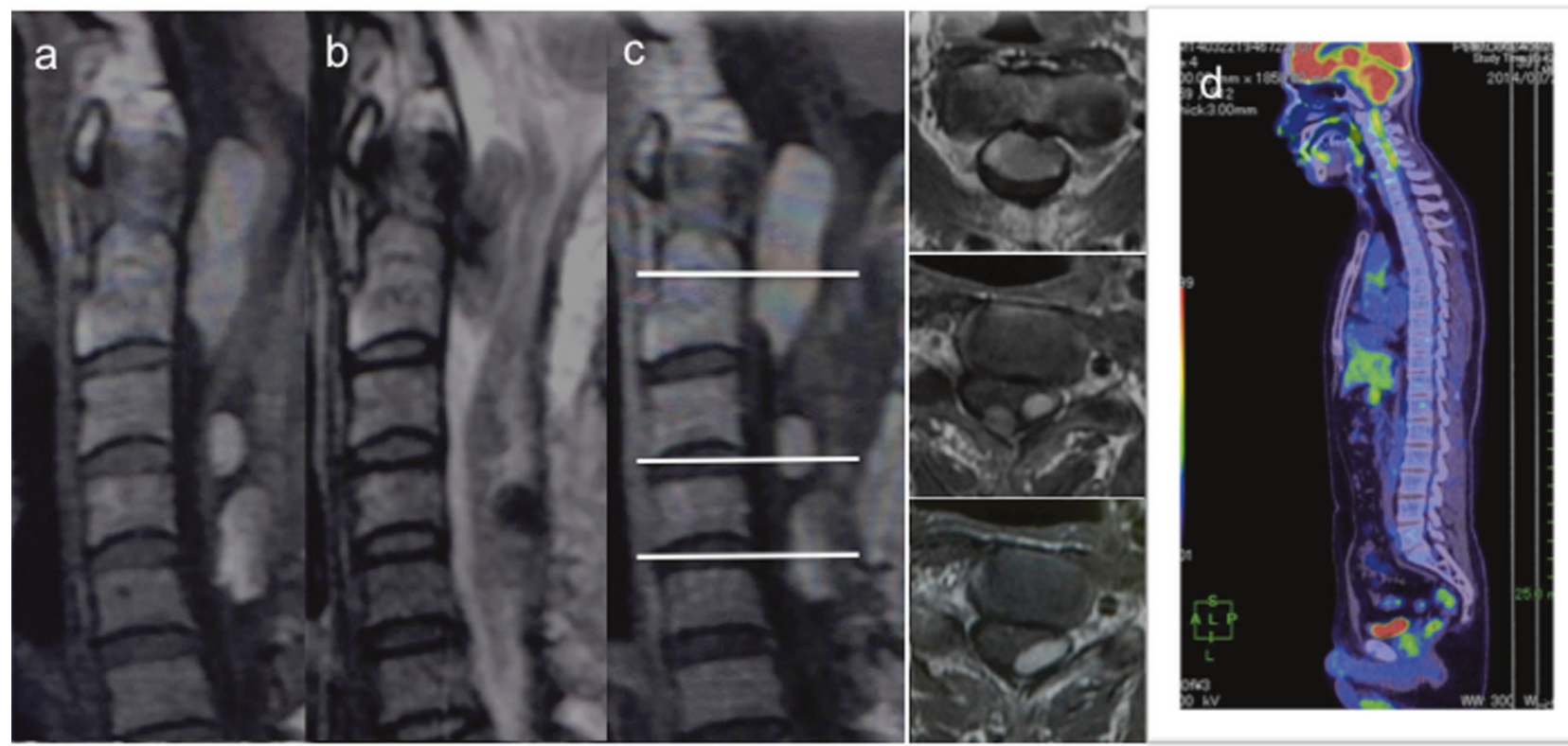

Fig. 1 a-c Magnetic resonance imaging shows several dura-based extramedullary solid tumors ventral to the $\mathrm{C} 1-2$ spinal cord and dorsal to the C3-4 and C4-C5 spinal cord. The tumors are hyperintense on T1-weighted images (a), isointense and hypointense on T2-weighted images (b), and strongly enhanced by gadolinium on T1-weighted

other lesions were noted on the whole spine image. We consulted a dermatologist, who was unable to find any skin lesion indicative of malignant melanoma. Brain MRI, positron emission tomography-computed tomography, gastroscopy, and colonoscopy were performed, none of which showed any clearly identifiable lesion (Fig. 1d). Therefore, we made a diagnosis of primary spinal cord tumor with suspected malignant melanoma. We then performed a diagnostic biopsy. Open-door laminoplasty was performed from $\mathrm{C} 2$ to $\mathrm{C} 5$ via a standard posterior cervical midline approach. The dura mater was almost entirely black, so we obtained a biopsy specimen from tissue that had spontaneously emerged from the dura mater (Fig. 2a). Histological analysis with hematoxylin-eosin staining revealed a highly cellular tumor that was composed of spindle cells arranged in fascicles with moderate nuclear atypia (Fig. 3a). No mitotic activity or necrosis was observed, and the MIB-1 labeling index was $<1 \%$ (Fig. 3b). Immunohistochemistry revealed that the tumor cells were positive for S100, HMB45, and Melan-A (Fig. 3c, d). On the basis of these findings, the pathological diagnosis was malignant melanoma. Tumorectomy was performed to prevent worsening of the patient's neurological symptoms.

We performed open-door laminoplasty and re-exposed the black-colored dura. The dura was incised, revealing a black well-encapsulated subdural tumor attached to the arachnoid mater (Fig. 2b). We dissected the tumor carefully from the surrounding structures and achieved near-total resection. The pathological diagnosis was unchanged. images (c). Axial images are gadolinium-enhanced T1-weighted tumors at $\mathrm{C} 1-2, \mathrm{C} 3-4$, and $\mathrm{C} 4-5$, respectively. d Positron emission tomography-computed tomography shows no clearly identifiable lesions except for those in the cervical spine
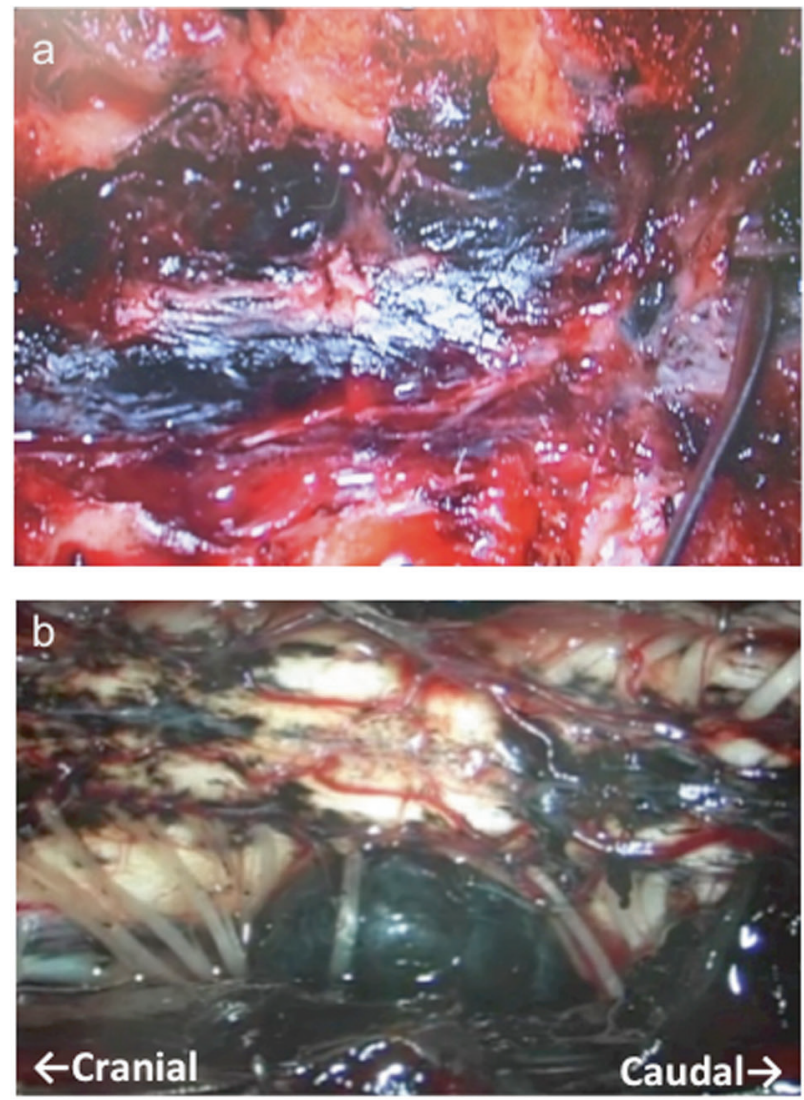

Fig. 2 a Intraoperative image of the black dura during the first biopsy procedure. b Intraoperative image of the black subdural wellencapsulated mass 
Fig. 3 a Highly cellular tumor composed of spindle cells arranged in fascicles, with moderate nuclear atypia.

Hematoxylin-eosin, $\times 400$. b Ki67 immunostaining. MIB-1 labeling index $<1 \%, \times 400$. c, d Tumor cells (yellow arrows) are positive for HMB-45 $(\times 400)(\mathbf{c})$ and Melan-A $(\times 400)(\mathbf{d})$
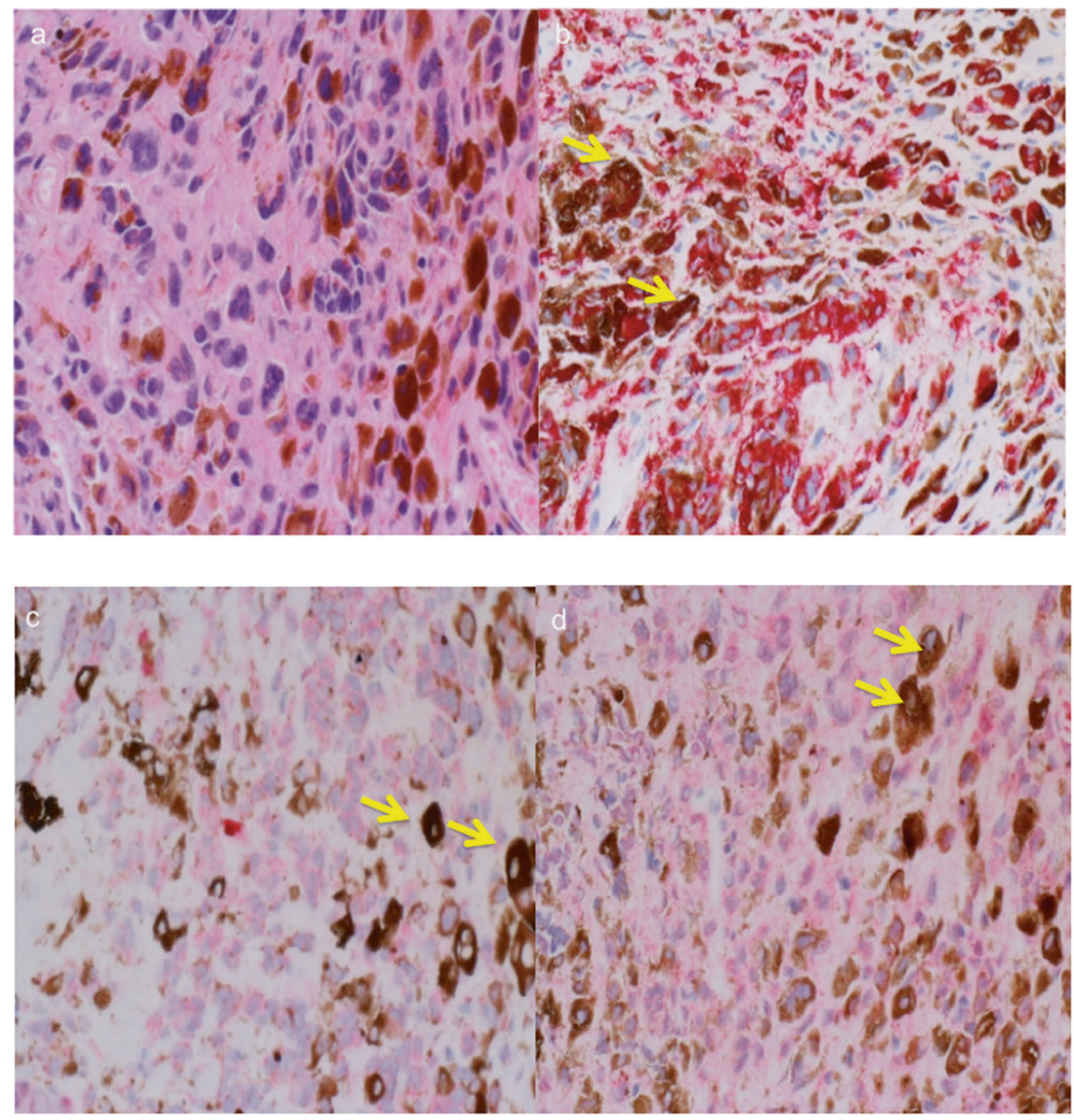

Postoperatively, the numbness and muscle weakness of the upper extremities improved and he was discharged with full motor strength, classified as AIS grade E at the level of C5.

The patient underwent chemotherapy (with anti-PD-1 antibody) but not radiotherapy after surgery. As of this writing ( 2 years postoperatively), follow-up MRI has shown no evidence of recurrence (Fig. 4).

The patient provided written informed consent for this case report to be published.

\section{Discussion}

Primary malignant melanoma of the central nervous system (CNS) is a very rare tumor, accounting for $\sim 1 \%$ of all cases of melanoma, and primary spinal cord melanoma is rarely encountered. Multifocal primary melanocytic tumor is extremely rare [2].

Primary melanoma of the spinal cord occurs most often at the thoracic level [3]. It is thought that primary malignant melanoma of the CNS originates in leptomeningeal melanoblasts, which are derived from the neural crest early in embryonic development [4]. Primary melanoma of the CNS is diagnosed using the criteria devised by Hayward as follows: no malignant melanoma outside the CNS; no presence of the lesion in any other region of the CNS; and confirmation of the melanoma histologically [5]. The World Health Organization classifies primary melanocytic tumors into meningeal melanomatosis, malignant melanoma, melanocytoma, and diffuse melanocytosis. Primary CNS melanoma shows positive staining for vimentin, S100 protein, and HMB-45. Melanocytoma is also a rare tumor arising from leptomeningeal melanocytes, but carries a better prognosis than melanoma. Melanocytoma is distinguished from melanoma by the presence or absence of necrosis, mitotic figures, nuclear atypia, microvascular invasion, high MIB-1 labeling index, and other features [6]. The MIB-1 labeling index indicates the proportion of cells positive for Ki-67 (a protein highly expressed in proliferating cells and encoded by the MIB-1 gene). Ki-67 immunoreactivity indicates proliferating cells. Thus, high MIB-1 labeling index suggests a high number of proliferating cells, which is 


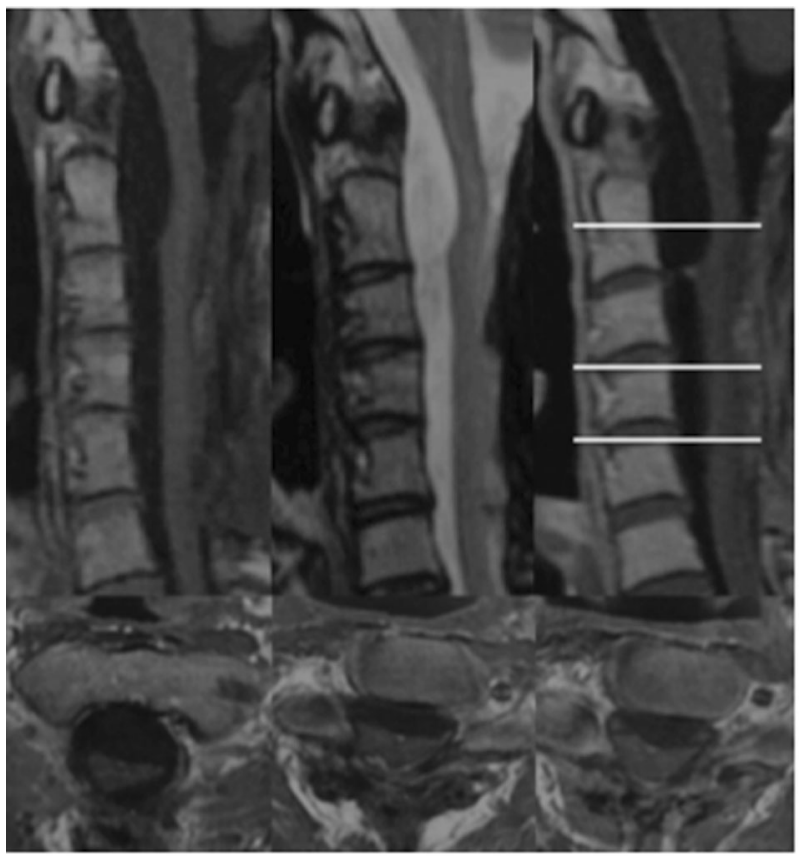

Fig. 4 Follow-up magnetic resonance imaging shows no evidence of recurrence at 2 years postoperatively. a, b T2-weighted image, b T2weighted image, $\mathbf{c}$ gadolinium-enhanced T1-weighted image

indicative of high malignant potential. MIB-1 labeling index is used as a tumor proliferation marker and is known to correlate with tumor grade and prognosis. Nevertheless, distinguishing between a malignant melanoma, a welldifferentiated melanocytoma, and an intermediate-grade melanocytic lesion [3] remains a diagnostic challenge. Hoffmann et al. [7] recently suggested that molecular analysis was the best method for distinguishing between melanocytoma and malignant melanoma.

MRI is by far the best diagnostic imaging procedure for spinal cord melanomas. These tumors are usually slightly hyperintense on T1-weighted images and isointense to hypointense on T2-weighted images [8]. However, the degree of intensity on T1/T2 depends on the individual tumor. Melanoma of the spinal cord is difficult to treat, with surgical resection appearing to yield the best outcome. Many reports recommend postoperative radiotherapy or chemotherapy; however, the efficacy of these adjuvant therapies is still controversial [9].

Mean survival time of patients who have undergone surgical excision of spinal cord melanoma with or without additional treatment is 6 years and 7 months after the onset of symptoms. In contrast, the mean survival time in patients with CNS metastases from melanoma of the skin is only 6 months [10]. The incidence of recurrence is greatly affected by tumor grade.
In this case, we performed near-total resection and achieved a favorable outcome, probably because the MIB-1 labeling index of the tumors was less than $1 \%$. We consider the 'primary multifocal' aspect of a melanocytic tumor to be highly suggestive of dissemination, especially in this case. Because we could not resect the black-colored dura mater completely, careful follow-up observation is still needed.

In summary, we have reported a rare case of multifocal primary melanoma of the cervical spinal cord. Near-total tumor resection was achieved, anti-PD-1 antibody chemotherapy was administered postoperatively, and no local recurrence has been observed in the 2 years after surgery. However, continued careful follow-up is deemed mandatory.

\section{Compliance with ethical standards}

Conflict of interest The authors declare that they have no conflict of interest.

\section{References}

1. Yu J, Zhao DD, Chen S, Zhang JM, Xu J. Primary melanoma of the cervical spine with cerebral metastases: case report and review of the literature. J Int Med Res. 2012;40:1207-15.

2. Wang YB, Wang WJ, Zhao HT, Li W, Peng T. Multiple melanocytoma of the thoracic spine: a case report and literature review. Spine J. 2016;16:59-63.

3. Huang X, Pan X, Huang H, Zhan R. Multiple spinal cord melanoma: case report with emphasis on the difficult preoperative diagnosis. Turk Neurosurg. 2013;23:534-8.

4. Pappenheim E, Bhattacharji SK. Primary melanoma of the central nervous system. Clinical-pathological report of a case, with survey and discussion of the literature. Arch Neurol. 1962;7:101-13.

5. Hayward RD. Malignant melanoma and the central nervous system. A guide for classification based on the clinical findings. J Neurol Neurosurg Psychiatry. 1976;39:526-30.

6. Reddy R, Krishna V, Sahu BP, Uppin M, Sundaram C. Multifocal spinal meningeal melanocytoma: an illustrated case review. Turk Neurosurg. 2012;22:791-4.

7. Hoffmann M, Koelsche C, Seiz-Rosenhagen M, Mai S, Lohr F, Reuss D, et al. The GNAQ in the haystack: intramedullary meningeal melanocytoma of intermediate grade at T9-10 in a 58year-old woman. J Neurosurg. 2015;6:1-4.

8. Farrokh D, Fransen P, Faverly D. MR findings of a primary intramedullary malignant melanoma: case report and literature review. Am J Neuroradiol. 2001;22:1864-6.

9. Patchell RA, Tibbs PA, Regine WF, Payne R, Saris S, Kryscio RJ, et al. Direct de-compressive surgical resection in the treatment of spinal cord compression caused by metastatic cancer: a randomised trial. Lancet. 2005;366:643-8.

10. Nishihara M, Sasayama T, Kondoh T, Tanaka K, Kohmura E, Kudo $\mathrm{H}$. Long-term survival after surgical resection of primary spinal malignant melanoma —case report. Neurol Med Chir. 2009;49:546-8. 